\title{
NOVAS TENDÊNCIAS NO TRATAMENTO DE EFLUENTES TÊXTEIS
}

\author{
Airton Kunz*, Patricio Peralta-Zamora \\ Departamento de Química, Universidade Federal do Paraná, CP 19081, 81531-990 Curitiba - PR \\ Sandra Gomes de Moraes e Nelson Durán \\ Instituto de Química, Universidade Estadual de Campinas, CP 6154, 13083-970 Campinas - SP
}

Recebido em 25/7/00; aceito em 6/6/01

\begin{abstract}
NEW TENDENCIES ON TEXTILE EFFLUENT TREATMENT. Textile effluents, when not correctly treated, cause a high impact to the environment. The main recalcitrant compounds present in textile effluent are represented by the synthetic dyes, used during the fibber dying process. Among others, the azo dyes are considered the most harmful due to its mutagenic and carcinogenic character. In the present work we reported a revision study on the new tendencies for remediation of textile effluents, mainly to degrade the recalcitrant compounds. For this purpose, chemical, physical, photochemical, biological and combined processes were investigated.
\end{abstract}

Keywords: textile effluents; treatment processes; recalcitrant compounds.

\section{INTRODUÇÃO}

Nas últimas décadas, os problemas ambientais têm se tornado cada vez mais críticos e freqüentes, principalmente devido ao desmedido crescimento populacional e ao aumento da atividade industrial. Com estes ingredientes os problemas devido a ação antrópica têm atingido dimensões catastróficas, podendo ser observadas através de alterações na qualidade do solo, ar e água.

Sem dúvida, a contaminação de águas naturais tem sido um dos grandes problemas da sociedade moderna. A economia de água em processos produtivos vem ganhando especial atenção devido ao valor agregado que tem sido atribuído a este bem, através de princípios como consumidor pagador e poluidor pagador recentemente incorporados em nossa legislação. Aliado a isso, temos previsões não muito animadoras para o século que se inicia, como por exemplo a previsão feita pela Companhia de Saneamento do estado de São Paulo (SABESP), que estima que já em 2010 a demanda de água será superior a capacidade hídrica dos mananciais do estado ${ }^{1}$.

Dentro deste contexto, o setor têxtil apresenta um especial destaque, devido a seu grande parque industrial instalado gerar grandes volumes de efluentes, os quais, quando não corretamente tratados, podem causar sérios problemas de contaminação ambiental.

Os efluentes têxteis caracterizam-se por serem altamente coloridos, devido à presença de corantes que não se fixam na fibra durante o processo de tingimento ${ }^{2}$.

A importância dos corantes para a civilização humana é evidente e bem documentada ${ }^{3}$. Os corantes sintéticos são extensivamente utilizados na indústria têxtil, gráfica, fotográfica e como aditivos em derivados de petróleo. Aproximadamente 10.000 diferentes corantes e pigmentos são usados industrialmente, o que representa um consumo anual de cerca de $7 \times 10^{5}$ tons no mundo ${ }^{4-6}$ e 26.500 tons somente no Brasil?.

A molécula do corante utilizada para tingimento da fibra têxtil pode ser dividida em duas partes principais, o grupo cromóforo e a estrutura responsável pela fixação à fibra.

*e-mail: airton@quimica.ufpr.br
Existem vários grupos cromóforos utilizados atualmente na síntese de corantes. No entanto, o grupo mais representativo e largamente empregado pertence a família dos azocorantes (Figura 1), que se caracterizam por apresentarem um ou mais grupamentos $-\mathrm{N}=\mathrm{N}$ ligados a sistemas aromáticos. Os azocorantes representam cerca de $60 \%$ dos corantes atualmente utilizados no mundo, sendo extensivamente utilizados no tingimento de fibras têxteis ${ }^{8}$.

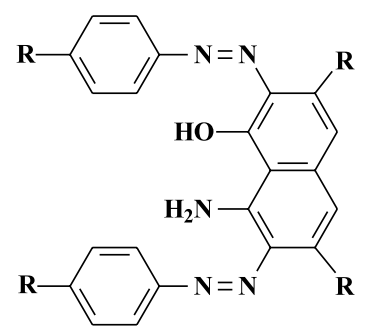

Figura 1. Exemplo de uma estrutura química característica de um grupo cromóforo de um azocorante.

A outra parte da molécula do corante, ligada ao grupo cromóforo, é responsável pela fixação do corante à fibra. Existem atualmente várias classes de corantes classificados segundo sua fixação, como por exemplo ácido, direto, básico, de enxofre e reativos, sendo este último o mais utilizado em nível mundial ${ }^{3,9}$.

Os corantes reativos são assim chamados devido a sua capacidade de formarem ligações covalentes com a fibra (Figura 2). Estes corantes podem ser utilizados no tingimento de fibras celulósicas com boas características de tingimento, solidez e estabilidade química $^{9,11}$

A poluição de corpos d'água com estes compostos provocam, além da poluição visual, alterações em ciclos biológicos afetando principalmente processos de fotossíntese. Além deste fato, estudos tem mostrado que algumas classes de corantes, principalmente azocorantes, e seus subprodutos, podem ser carcinogênicos e/ou mutagênicos ${ }^{12-14}$.

Devido a estas implicações ambientais, novas tecnologias têm sido buscadas para a degradação ou imobilização destes compostos em efluentes têxteis. 


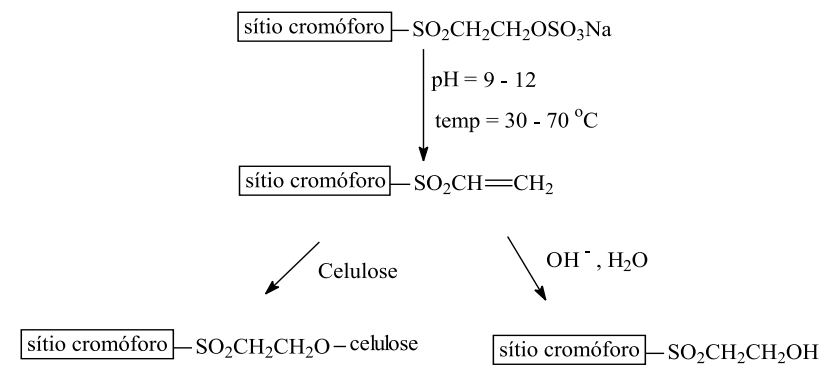

Figura 2. Interação de corantes reativos do tipo vinil sulfonato com a fibra têxtil ${ }^{10}$.

\section{PROCESSOS DE TRATAMENTO UTILIZADOS PELA INDÚSTRIA TÊXTIL}

As técnicas de tratamento fundamentadas em processos de coagulação, seguidos de separação por flotação ou sedimentação, apresentam uma elevada eficiência na remoção de material particulado. No entanto, a remoção de cor e compostos orgânicos dissolvidos mostram-se deficientes. Os processos de adsorção em carvão ativado apresentam uma eficiência significativamente maior, contudo em função da superfície química do carvão ser positiva, a adsorção de corantes de caráter catiônico é uma limitação bastante importante ${ }^{15}$.

Além das desvantagens aqui assinaladas, é importante salientar que todos os processos anteriormente citados correspondem a sistemas não destrutivos. Embora o volume dos resíduos possa ser significativamente diminuído, a disposição final das fases sólidas continua sendo um problema sem solução.

Em função destes inconvenientes, existe uma certa predileção pela utilização de processos que realmente possam degradar as espécies de interesse. Dentro do contexto dos processos destrutivos, cabe aos processos biológicos um lugar de destaque, principalmente em função da relativa facilidade encontrada na implementação de sistemas que operem em grande escala.

Os processos biológicos utilizados com maior frequiência estão representados pelos sistemas de lodos ativados (Figura 3). Este processo consiste na agitação dos efluentes na presença de microorganismos e ar, durante o tempo necessário para metabolizar e flocular uma grande parte da matéria orgânica ${ }^{17}$. Infelizmente, o processo apresenta o grande inconveniente de ser bastante susceptível à composição do efluente (cargas de choque), além de produzir um grande volume de lodo.

Em geral, na indústria têxtil os processos de tratamento estão fundamentados na operação de sistemas físico-químicos de precipitação-coagulação, seguidos de tratamento biológico via sistema de lodos ativados. O sistema apresenta uma eficiência relativamente alta, permitindo a remoção de aproximadamente $80 \%$ da carga de corantes. Infelizmente, o problema relacionado com o acúmulo de lodo tornase crítico, uma vez que o teor de corantes adsorvido é bastante elevado, impedindo qualquer possibilidade de reaproveitamento.

Por todos estes motivos, o estudo de novas alternativas para o adequado tratamento de efluentes deve ser considerado como uma prioridade dos profissionais que atuam nesta área de trabalho.

\section{ÚTLIMOS AVANÇOS NO TRATAMENTO DE CORANTES E EFLUENTES TÊXTEIS}

\section{Biodegradação}

A grande motivação de todos os pesquisadores envolvidos em estudos de biodegradação pode ser expressa pela busca contínua de

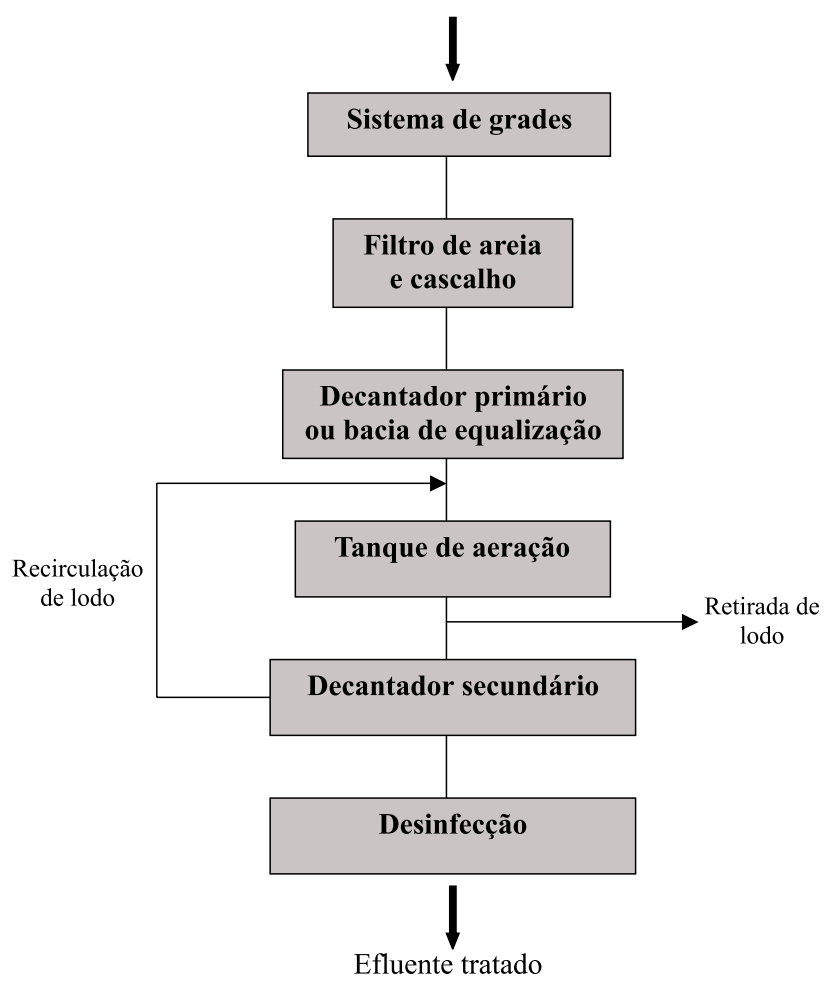

Figura 3. Esquema de uma estação de tratamento de efluentes utilizando lodo ativado, tipicamente empregada para tratamento de efluente têxtil ${ }^{16}$.

microrganismos versáteis, capazes de degradar de maneira eficiente um grande número de poluentes a um baixo custo operacional. $\mathrm{Na}$ prática, sabemos que isto é muito difícil principalmente em função da diversidade, concentração e composição de espécies químicas presentes em cada efluente.

Recentemente, pesquisadores tem aumentado o interesse no versátil fungo de decomposição branca Phanerochaete chrysosporium. Este fungo tem a capacidade de mineralizar, além da lignina, pelo menos parcialmente e em alguns casos completamente, uma variedade de poluentes resistentes a degradação ${ }^{18-21}$.

O sistema lignolítico deste fungo é representado principalmente pelas enzimas lignina e manganês peroxidase, as quais são produzidas em meios contendo fontes limitadas de carbono e nitrogênio. Estas enzimas tem a capacidade de despolimelizar a lignina e uma grande variedade de outros compostos.

Especialmente para efluentes têxteis, alguns estudos tem sido realizados explorando sua capacidade de degradar e mineralizar corantes. Spadaro e col. ${ }^{4}$ demonstraram que $P$. chrysosporium foi capaz de mineralizar alguns azocorantes, sendo a capacidade de descoloração diretamente relacionada com a natureza dos grupos substituintes dos anéis aromáticos. Kirby e $\mathrm{col}^{22}$ estudando a capacidade de descoloração deste fungo frente a uma amostra de efluente simulada em laboratório, observaram a descoloração total deste após

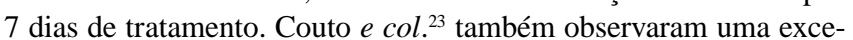
lente eficiência no tratamento de uma amostra contendo o corante poli-R-478, alcançando descolorações superiores a $95 \%$ após o tratamento com o fungo $P$. chrysosporium.

A utilização de outros fungos como por exemplo Pleorotus ostreatus e Trametes versicolor para degradação de corantes também vem sendo estudada ${ }^{24-27}$. Estes fungos caracterizam-se por serem bons produtores de lacase, uma fenol oxidase dependente de cobre. Esta enzima lignolítica tem a capacidade de catalisar reações de desmetilação, que é um inicial e importante passo em processos 
de biodegradação de cadeias poliméricas, com subsequente decomposição de macromoléculas de lignina pelo rompimento de anéis aromáticos e ligações $\mathrm{C}_{\mathrm{a}}-\mathrm{C}_{\mathrm{b}}$ em estruturas fenólicas. Em processos de oxidação de muitos compostos (principalmente de compostos fenólicos) a lacase apresenta uma grande especificidade para um grande número de compostos xenobióticos e efluentes industriais ${ }^{28}$.

A utilização de bactérias, como Pseudomonas $s p^{29-31} \mathrm{e}$ Sphingomonas $s p^{32-34}$ tem sido reportadas na degradação de corantes. Estes microrganismos são particularmente úteis para degradação de azocorantes, pois tem a capacidade de realizar a clivagem redutiva nas ligações azo deste tipo de composto, fato este que geralmente está associado a enzima azoredutase.

Outra alternativa recente para o tratamento de efluentes e compostos resistentes à degradação refere-se ao uso de agentes quelantes naturais, produzidos por alguns fungos e bactérias. Estes compostos, denominados sideróforos, apresentam uma alta afinidade por metais, principalmente ferro, formando complexos de alta estabilidade. A função biológica dos sideróforos é seqüestrar ferro em ambientes com deficiência deste ${ }^{35}$.

Existem basicamente dois tipos de sideróforos caracterizados até o momento, o do tipo catecolato e o do tipo hidroxamato. A presença do tipo catecolato foi detectada em vários fungos lignolíticos ${ }^{36-40} \mathrm{o}$ que não foi tão freqüentemente observado com o tipo hydroxamato ${ }^{41-43}$. Estudos recentes mostram resultados muito interessantes com alguns fungos e bactérias produtoras destes compostos, demonstrando uma atividade mimética de fenoloxidase, o que permitiu a descoloração de efluentes e a degradação de compostos de difícil degradação ${ }^{44-46}$.

\section{Tratamento com ozônio}

Ozônio, a forma triatômica do oxigênio, é um gás incolor de odor pungente. Em fase aquosa, o ozônio se decompõe rapidamente a oxigênio e espécies radicalares. $\mathrm{O}$ ozônio é um agente oxidante poderoso $\left(\mathrm{E}_{0}=2,08 \mathrm{~V}\right)$ quando comparado a outros agentes oxidantes conhecidos como por exemplo $\mathrm{H}_{2} \mathrm{O}_{2}\left(\mathrm{E}_{0}=1,78 \mathrm{~V}\right)$ permitindo com que esta espécie reaja com uma numerosa classe de compostos ${ }^{47-49}$.

A oxidação de poluentes ou efluentes pode ocorrer de maneira direta ou indireta ${ }^{47,50}$.

\section{Oxidação direta}

Através deste processo a molécula de ozônio pode reagir diretamente com outras moléculas orgânicas ou inorgânicas via adição eletrofílica (Figura 4). O ataque eletrofílico do ozônio pode acontecer a átomos com uma densidade de carga negativa (N, P, O ou carbonos nucleofílicos) ou a ligações duplas ou triplas do tipo carbonocarbono, carbono-nitrogênio e nitrogênio-nitrogênio ${ }^{51,52}$.

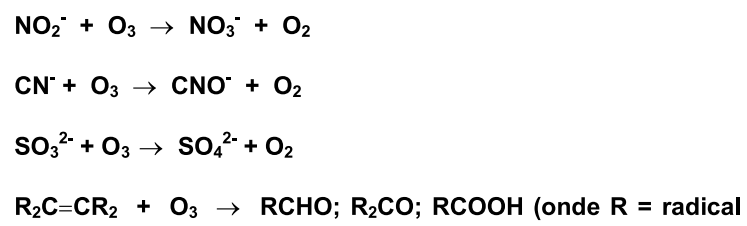

Figura 4. Alguns exemplos de oxidação direta com ozônio ${ }^{48}$.

\section{Oxidação indireta}

Indiretamente, o ozônio pode reagir através de reação radicalar (principalmente $\cdot \mathrm{OH}$ ) que é gerado pela decomposição do ozônio.
(Figura 5). O radical hidroxila é um poderoso e não seletivo oxidante $\left(\mathrm{E}^{\mathrm{o}}=2,80 \mathrm{~V}\right)$ que pode reagir através de três mecanismos distintos: i)abstração de hidrogênio ii) transferência de elétrons ou iii) adição radicalar (Figura 6). Os radicais secundários formados durante estas reações podem novamente reagir com ozônio ou outros compostos.

$$
\begin{aligned}
& \mathrm{O}_{3}+\mathrm{H}_{2} \mathrm{O} \stackrel{h v}{\longrightarrow} \mathrm{H}_{2} \mathrm{O}_{2}+\mathrm{O}_{2} \\
& \mathrm{H}_{2} \mathrm{O}_{2} \stackrel{h v}{\longrightarrow} 2 \cdot \mathrm{OH} \\
& \mathrm{O}_{3}+\mathrm{OH}^{-} \longrightarrow \mathrm{O}_{2}^{-}+\mathrm{HO}_{2}{ }^{\circ} \\
& \mathrm{O}_{3}+\mathrm{HO}_{2} \cdot 2 \mathrm{O}_{2}+\cdot \mathrm{OH}
\end{aligned}
$$

Figura 5. Reações de decomposição de ozônio formando espécies radicalares $^{48,53}$.

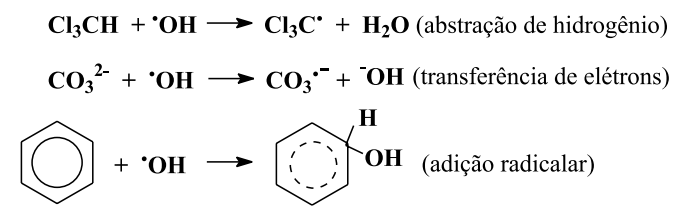

Figura 6. Reações iniciadas por radical hidroxila ${ }^{54}$.

Para o tratamento de efluente têxtil o ozônio se mostra muito atrativo. Geralmente, os cromóforos encontrados neste efluente são compostos orgânicos com grande conjugação de ligações duplas como mencionado acima. Estas ligações podem ser rompidas por ozônio (direta ou indiretamente) formando moléculas menores descolorindo assim o efluente.

Neste sentido, vários estudos tem sido realizados demonstrando a eficiência do ozônio na remoção de cor em efluentes têxteis. Lin e Liu $^{55}$ observaram a descoloração de efluentes têxteis com ozônio aplicado em contra-corrente em tempos usualmente menores que 5 minutos. Shu e Huang ${ }^{56}$ estudando uma mistura de oito azocorantes e Liakou e Liberatos ${ }^{57}$ estudando a degradação do azocorante laranja 2, também observaram uma rápida degradação destes.

Kunz e $c l^{58}$ e Peralta-Zamora e $c l^{59}$ estudando a descoloração de alguns corantes reativos e Kunz e $\operatorname{col}^{60}$ estudando a degradação de efluente têxtil também observaram uma efetiva e rápida descoloração das amostras com ozônio.

No entanto, um inconveniente muitas vezes encontrado nos estudos de degradação com ozônio refere-se ao aumento da toxicidade de alguns intermediários de reação ${ }^{58,61}$, o que torna necessário o acompanhamento do processo através de testes de toxicidade.

\section{Fotocatálise heterogênea}

A degradação de compostos orgânicos através de fotocatálise heterogênea, assim como os princípios que fundamentam o processo, têm sido bastante documentados ${ }^{62-64}$.

Conforme representado na Figura 7, quando um semicondutor é exposto a luz ultravioleta, este é promovido a um estado eletronicamente excitado que se caracteriza pela geração de um par elétronlacuna $\left(\mathrm{e}^{-}, \mathrm{h}^{+}\right)$. As características oxidantes do radical hidroxila gerado por reação da lacuna (equação 1), e o caráter fortemente oxidante da própria lacuna, fazem com que as moléculas orgânicas adsorvidas na superfície da partícula do catalisador possam ser oxidadas até completa mineralização, através de um processo bastante viável.

$(\mathrm{Ti})_{\text {superfície }}-\mathrm{OH}+\mathrm{h}^{+} \rightarrow(\mathrm{Ti})_{\text {superfície }}-\mathrm{OH}^{*} \quad$ (Equação 1) 


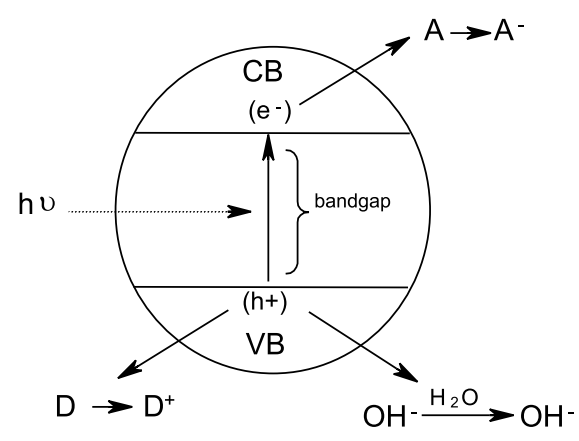

Figura 7. Representação esquemática dos princípios da fotocatálise heterogênea (A: Espécies aceptora, B: Espécies doadora).

A aplicação da fotocatálise no tratamento de efluentes têxteis tem sido relativamente pouco estudada, no entanto vários artigos tem sido publicados estudando-se a degradação de corantes isolados ${ }^{59,65-68}$.

Embora a elevada eficiência da fotocatálise heterogênea permita uma rápida mineralização de inúmeras espécies químicas de relevância ambiental, existem vários inconvenientes de ordem prática que tem dificultado bastante a sua consolidação como alternativa de tratamento em grande escala. Dentre as mais importantes limitações contam-se: 1). necessidade de fontes artificiais de radiação, uma vez que grande parte dos fatocatalisadores apresentam um "band gap" correspondente à região ultravioleta, 2). dificuldades na penetração da radiação no meio de reação e dificuldades na separação dos fotocatalisadores, uma vez que estes são utilizados na forma de finas suspensões e 3). dificuldades na implementação de sistemas contínuos em grande escala, principalmente em função dos inconvenientes anteriores.

Muitos estudos tendentes a contornar os inconvenientes acima citados estão sendo realizados no momento. Dentre as várias alternativas estudadas destacam-se: 1). Desenvolvimento de fotocatalisadores de maior eficiência (dopagem), 2). implementação de sistemas que operem com fotocatalisadores imobilizados e 3). utilização de fotocatalisadores passíveis de ativação por luz solar ou de sistemas assistidos por agentes sensibilizadores ${ }^{69-74}$.

\section{Processos físicos}

Dentre os processos físicos mais utilizados no tratamento de efluentes e corantes têxteis, a adsorção com carvão ativado ainda vem sendo intensamente estudada ${ }^{15,75-77}$. O estudo de alguns agentes alternativos utilizando-se de biomassa como adsorvente também tem despertado atenção recentemente. Alguns artigos tem sido publicados nos últimos anos utilizando carvão ativado de $\operatorname{coco}^{78}$, bambo ${ }^{79}$, casca de eucalyptus ${ }^{80}$ e quitosana ${ }^{81,82}$ como materiais adsorventes.

A utilização de tecnologias de membranas ${ }^{83-86}$ (Tabela 1), como osmose reversa (OR), microfiltração (MF), nanofiltração (NF) e ultrafitração (UF), têm se tornado muito atrativas devido ao fato de possibilitarem o reuso da água no processo industrial. Isto é especialmente interessante se analisarmos as perspectivas futuras não muito animadoras de escassez, elevação dos custos para captação de água e legislação cada vez mais restritiva para emissão de efluentes.

\section{Processos Combinados}

Para o tratamento de um dado efluente muitas vezes uma solução bastante inteligente é a utilização de processos combinados para uma melhor eficiência do sistema. Estes métodos podem ser utilizados de maneira complementar, de tal forma que possam suprir deficiências apresentadas pelos processos quando aplicados isoladamente.
Tabela 1. Características de alguns processos de separação por membrana com aplicações industriais ${ }^{87,88}$.

\begin{tabular}{lccc}
\hline $\begin{array}{c}\text { Processo } \\
\text { de separação }\end{array}$ & $\begin{array}{c}\text { Pressão } \\
\text { aplicada } \\
\text { (atm) }\end{array}$ & $\begin{array}{c}\text { Tamanho } \\
\text { do poro } \\
\text { (nm) }\end{array}$ & $\begin{array}{c}\text { Material } \\
\text { retido }\end{array}$ \\
\hline $\begin{array}{c}\text { Microfiltração } \\
\text { (MF) }\end{array}$ & $1-3$ & $20-1000$ & $\begin{array}{c}\text { material em } \\
\text { suspensão, } \\
\text { bactérias } \\
\text { (m.m. > 500.000) }\end{array}$ \\
\hline $\begin{array}{c}\text { Ultrafiltração } \\
\text { (UF) }\end{array}$ & $2-7$ & $5-20$ & $\begin{array}{c}\text { colóides, } \\
\text { macromoléculas } \\
\text { (m.m. > 5000) }\end{array}$ \\
\hline $\begin{array}{c}\text { Nanofiltração } \\
\text { (NF) }\end{array}$ & $5-20$ & $2-5$ & $\begin{array}{c}\text { macromoléculas } \\
\text { Osmose reversa } \\
\text { (OR) }\end{array}$ \\
\hline
\end{tabular}

Atualmente dispomos de vários métodos para tratamento de efluentes, podendo ser classificados principalmente em físicos, químicos e biológicos. A combinação destes para tratamento de um dado efluente vai depender muito dos objetivos que se quer atingir no tratamento ${ }^{89}$.

Para o tratamento de efluentes têxteis, a combinação de métodos mostra-se mais adequada, devido à presença de corantes que normalmente são resistentes a degradação nos sistemas convencionais de tratamento. Em geral, maior ênfase tem sido dado ao estabelecimento de metodologias que combinam os processos biológicos com outras alternativas físicas ou físico-químicas, tais como floculação, adsorção ou oxidação eletroquímica ${ }^{8,90}$.

A combinação de processos oxidativos avançados (POAs) utilizando-se peróxido de hidrogênio, ozônio, luz ultravioleta, $\mathrm{TiO}_{2}$ tem sido testados recentemente como alternativas aos processos de tratamento estabelecidos atualmente, com resultados promissores ${ }^{91-93}$. Processos físicos utilizando-se tecnologias de membranas combinadas principalmente com ozônio também tem recebido especial atenção no final da década de 90 devido a possibilidade de reuso da água conforme já discutido anteriormente ${ }^{94,95}$. A combinação de processos biológicos (anaeróbios-aeróbios) ${ }^{96}$ também é útil, pois permite a efetiva descoloração do efluente principalmente quando azocorantes estiverem presentes neste efluente. Fungos, principalmente os decomposição branca, em combinação com métodos biológicos e químicos ${ }^{97,98}$ também têm sido testados e se mostrado bastante eficientes na descoloração de efluentes e corantes têxteis.

\section{CONSIDERAÇÕES FINAIS}

O aumento da complexidade e dificuldade para o tratamento de efluentes têxteis e industrias de um modo geral, tem levado a busca constante de novas metodologias para tratamento destes rejeitos. Dispomos de uma variedade de métodos físicos, químicos e biológicos e a escolha do melhor, ou melhores, métodos seguramente deve ser feita levando-se em conta os objetivos a serem alcançados com o tratamento.

Aliado a isso, uma visão moderna com relação a efluentes industrias deve estar baseada não somente no tratamento deste (tecnologias "end of pipe") e sim na busca constante da minimização de resíduos gerados através de tecnologias limpas, ou seja, o pensamento deve se voltar para a fonte do efluente dentro da fábrica e não somente como resolver o problema após sua geração. 


\section{AGRADECIMENTOS}

\section{FAPESP, CNPq.}

\section{REFERÊNCIAS}

1. Furtado, M.R.; Quim. Der. 1997, março, 10.

2. O’Neill, C.; Hawkes, F.R.; Hawkes, D.L., Lourenco, N.D.; Pinheiro, H.M.; Delee, W.; J. Chem. Technol. Biotechnol. 1999, 74, 1009.

3. Encyclopedia of Chemical Technology. Vol 8 (third ed.). Ed. John Wiley \& Sons 1990.

4. Spadaro, J. T.; Gold, M. H.; Renganathan, V.; Appl. Environ. Microbiol. 1992, 58, 2397.

5. Nigam, P.; Marchant, R.; Biotech. Lett. 1995, 17, 993.

6. Nigam, P.; Banat, I. M.; Singh, D.; Marchant, R.; Process. Bioch. 1996, 31,435 .

7. Guaratini, C.C.I; Zanoni, M.V.B.; Quim. Nova 2000, 23, 71.

8. Vandevivere, P.V.; Bianch, R.; Verstraete, W.; J. Chem. Technol. Biotechnol. 1998, 72, 289.

9. Mangold, J.; Calquim. 1995, 34.

10. Weber, E.J.; Stichney, V.C.; Wat. Res. 1993, 27, 63

11. Alcantara, M.R.; Daltin, D.; Quim. Nova 1996, 19, 320.

12. Houk, V. S.; Wat. Res. 1992, 277, 91.

13. Chung, K-T; Cerniglia; C.E.; Mut. Res. 1992, 277, 201

14. Brown, M. A.; Devito, S.C.; Crit. Rev. Environ. Sci. Technol. 1993, 23, 249.

15. Al-Degs, Y.; Khraisheh, M.A.M.; Allen, S.J.; Ahmad, M.N.; Wat. Res. 2000, $34,927$.

16. Metcalf \& Eddy; Wastewater Engineering, Treatment, Disposal, Reuse. Ed. McGraw Hill, Third Ed., 1991.

17. Bitton, G. Wastewater Microbiology; ed. Willey-Liss, New York, 1994.

18. Paszczynski, A.; Pasti-Grigsby, M. B.; Goszczynski, S.; Crawford, D. L.; Crawford, R. L.; Enzime Microb. Technol. 1991, 13, 378.

19. Barr, D. P.; Aust, S. D.; Environ. Sci. Technol. 1994, $28,79$.

20. Duran, N.; Esposito, E.; em Microbiologia Ambiental (Melo, I.S.; Azevedo, J.L. - Embrapa-CNPMA; Jaguariuna-SP), 1997, 269.

21. Manimekalai, R.; Swaminathan, T.; Bioprocess. Eng. 2000, 22, 29.

22. Kirby, N.; Mullan, G. Mc; Marchant, R.; Biotech. Lett. 1995, 17, 761.

23. Couto S.R.; Rivela I.; Munoz M.R.; Sanroman A; Biores. Technol. 2000, $74,159$.

24. Abadulla E; Robra K.H.; Gubitz G.M.; Silva L.M.; Cavaco-Paulo, A.; Text. Res. J. 2000, 70, 409.

25. Wong, Y.X.; Yu. J.; Wat. Res. 1999, 33, 3512.

26. Swamy, J.; Ramsay, J.A.; Appl. Microbiol. Biotechnol. 1999, 51, 391.

27. Rodríguez, E.; Pickard, M.A.; Vazquez-Duhalt, R.; Curr. Microbiol. 1999, $38,27$.

28. Duran, N.; Espósito, E.; Appl. Catal. B: Environ. 2000, 714, 1.

29. Sairnak, S.; Kanekar, P.; Appl. Microbil. Biotehcnol. 1999, 52, 251.

30. Hu, T.L.; Wat. Sci. Technol. 1998, 38, 299

31. Zissi, U.; Lyberatos, G.; Pavlou, S.; J. Ind. Microbiol. Biotechnol. 1997, 19, 49.

32. Russ, R.; Rau, J.; Stolz, A.; Appl. Environ. Microbiol. 2000, 66, 1429.

33. Coughlin , M.F.; Kinkle B.K.; Bishop, P.L.; J. Ind. Microbiol. Biotechnol. 1999, 23, 341 .

34. Kudlich, M.; Keck A.; Klein J.; Stolz, A.; Appl. Environ. Microbiol. 1997, 63, 3691.

35. Machuca, A.; Aoyama, H.; Duran, N.; Bichem. Biophys. Res. Com. 1999, 256, 20.

36. Duran, N.; Proc. 4th Braz. Symp. Chem. Lignins Wood Comp. 1995, 164.

37. Goodell, B.; Jellison, J.; Liu, J.; Daniel, G.; Paszcnski, A.; Fekete, F.; Krishnamurthy, S.; Jun, L.; Xu, G.; J. Biotechnol. 1997, 53, 133.

38. Rodriguez, J.; Oses, R.; Parra, C.; Freeer, J.; Baeza, J.; Proc. $5^{\text {th }}$ Braz. Symp. Chem. Lignin and Other Wood Comp. 1997, 601.

39. Parra, C.; Rodriguez, J.; Baeza, J.; Durán, N.; Proc. $5^{\text {th }}$ Braz. Symp. Chem. Lignin Wood Comp. 1997, 528

40. Parra, C.; Santiago, M.F.; Rodriguez, J. Durán, N.; Biochem. Biophys. Res. Commun. 1998, 249, 719.

41. Durán, N.; Machuca, A.; Holz als Roh und Werstoff 1995, 53, 346.

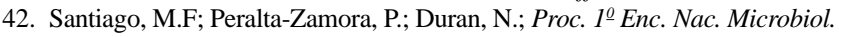
Apl. Meio Amb. 1997, 174.

43. Santiago, M.F.; Peralta-Zamora, P.; Duran, N.; Proc. $5^{\text {th }}$ Braz. Symp. Chem. Lignin Other Wood Comp. 1997, 567.

44. Duran; N. Parra, C.; Santiago, M.F.; Rodriguez, J.; Proc. $5^{\text {th }}$ Europ. Workshop Lignocellul. Pulp. 1998, 83.

45. Durán, N.; Santiago, M.F.; Rodriguez, J.; Parra, C.; Oses, R.; Freer, J.; Baeza, J.; Proc. 10 th Intern. Symp. Wood and Pulping Chem. 1999, 286.

46. Oses, R.; Rodriguez, J.; Tasumi, K.; Freer, J.; Baeza, J.; Proc. $7^{\text {th }}$ Intern. Conf. Biotechnol. Pulp Paper Ind. 1998, 227.
47. Masten, S.T.; Davies, S.H.R.; Environ. Sci. Technol. 1994, 28, 181A.

48. Chamarro, E.; Marco, A.; Prado, J.; Esplugas, S.; Quim. Ind. 1996, 1/2, 28.

49. Kunz, A.; Freire, R.S.; Rowedler, J.J.R.; Mansilla, H.; Rodriguez, J.; Duran, N.; Quim. Nova 1999, 22, 425.

50. Kunz, A., Tese de Doutorado, Instituto de Química-Unicamp, 1999.

51. Lin, S.H.; Yeh, K.L.; Chem. Eng. 1993, May, 112

52. Esplugas, S., Curso técnico, 8-10 de novembro, 1995, LRR-Universidad de Concepcion-Chile.

53. Peleg,M.; Wat. Res. 1976, 10, 361 .

54. Calvosa, L.; Monteverdi, A.; Rindone, B; Riva, G.; Wat. Res. 1991, 25, 985

55. Lin. S. H.; Liu, W. Y.; Environ. Technol. 1994, 15, 299

56. Shu, H.; Huang, C.; Chemosphere 1995, 31, 3813.

57. Liakou, S. Libertatos, G., Proc. Int. Conf.- Oxid. Technol. Wat. Wastewat. Treat. - ClausthalerUm-welttechnik-Institut GmH, German, 1996.

58. Kunz, A.; Mansilla, H. Freer, J. Baeza, J.; Duran, N.; Proc. $5^{\text {th }}$ Braz. Symp. on the Chem. Lignins Wood Compon. 1997, 92.

59. Peralta-Zamora, P.; Kunz, A.; Moraes, S.G.; Pelegrini, R.; Moleiro, P.C.; Reyes, J.; Durán, N.; Chemosphere 1999, 38, 835.

60. Kunz, A.; Reyes, J.; Durán, N. ; Proc. Int. Conf. Dif. Poll., 1999, 16-20 may. Perth-WA-Australia.

61. Langlais, B.; Legube, B.; Beuffe, H.; Doré, M.; Wat. Sci. Technol. 1992, 25,135 .

62. Legrini, A.; Oliveros, E.; Braun, A. M.; Chem. Rev. 1993, 93, 671.

63. Linsenbigler, A. L.; Guangquan, L; Yates, Jr. T.; Chem. Rev. 1995, 95, 735.

64. Hoffmann, M.; Martin, S.T.; Choi, W; Bahnemann, W.; Chem. Rev. 1995, $95,69$.

65. Vinodgopal, K; Wynkoop, D.E.; Kamat, P.V.; Environ. Sci. Technol. 1996, 30,1660 .

66. Tanaka, K.; Padermpole, K.; Hisanaga, T.; Wat. Res. 2000, 34, 327.

67. Poulios, I.; Aetopoulou, I.; Environ. Technol. 1999, $20,479$.

68. Wang, Y.Z.; Wat. Res. 2000, 34, 990.

69. Pelegrini, R.; Peralta-Zamora, P.; Andrade, A.P.; Reyes, J.; Duran, N.; Appl. Catal. B: Environ. 1999, 566,1.

70. Gouvêa, C,A,K; Wypych, F.; Moraes, S,G; Durán, N.; Nagata, N. PeraltaZamora, P.; Chemosphere 2000, 40, 433.

71. Kiriakidou, F.; Kondarides, D.I.; Verykios, X.E.; Catal. Today 1999, 54, 119

72. Li, X.Z.; Zhao, Y.G.; Wat. Sci Technol.1999, 39, 249.

73. Naskar, S.; Pillay, S.A.; Chanda, M.; J. Photochem. Photobiol. A. 1998, $113,257$.

74. Zhu, C.M.; Wang, L.Y.; Kong L.R.; Yang, X.; Wang, L.S.; Zheng, S.J.; Chen, F.L.; Feng M.Z; Zong, H.; Chemosphere 2000, 41, 303.

75. Nicolet, L.; Rott, U.; Wat. Sci Technol. 1999, 40, 191.

76. Choy, K.K.H.; McKay, G.; Porter, J.F.; Res. Conserv. Rec. 1999, $27,57$.

77. Danis, U.; Gurses, A.; Canpolat. N.; Fresenius Environ. Bull.1999, 8, 358.

78. Kadirvelu, K.; Palanival, M.; Kalpana, R.; Rajeswari, S.; Biores. Technol. 2000, 74, 263

79. Wu, F.C.; Tseng, R.L.; Juang, R.S.; J. Environ. Sci. Heal. A 1999, 34, 1753.

80. Morais, L.C.; Freitas, O.M.; Gonçalves, E.P.; Vasconcelos, L.T.; Beca, C.G.G.; Wat. Res. 1999, 33, 979

81. Juang, R.S.; Tseng, R.L.; Wu, F.C.; Lee, S.H.; J. Chem. Technol. Biotechnol. 1997, 70, 391

82. No, H.K.; Meyers, S.P.; Rev. Environ. Contam. Toxicol. 2000, 163; 1

83. Porter, J.J.; Gomes, A.C.; Desalination 2000, 128, 81.

84. Reife, A.; Freeman, H.S.; Text. Chem. Color. Am. Dyes. Rep. 2000, $32,56$.

85. Sojka-Ledakowicz, J.; Koprowski, T.; Machnowski, W.; Knudsen, H.H. Desalination 1998, 119, 1.

86. Diaper, C.; Correia, V.M.; Judd, S.J. ; J. Soc. Dyers Colour 1996, 112, 273.

87. Madaeni, S.S. ; Wat. Res. 1999, 33, 301.

88. Nobrega, R.; An.II Semin. Int. Tecnol. Enz., Rio de Janeiro - RJ, 1995.

89. Esplugas, S.; Marco, A.; Saum, G.; Proc. Int. Conf.- Oxid. Technol. Wat. Wastewat. Treat. - ClausthalerUm-welttechnik-Institut GmH, German, 1996.

90. Lin, S. H.; Peng, C. F.; Wat. Res. 1996, 30, 587.

91. Arslan, I.; Balcioglu, I.A.; Tuhkanen, T.; Environ. Technol. 1999, 20, 921.

92. Galindo, C.; Jacques, P.; Kalt, A.; J. Photochem. Photobiol. A 2000, 130, 35.

93. de Moraes, S.G.; Freire, R.S.; Duran, N.; Chemosphere 2000, 40, 369.

94. Wu, J.N.; Eiteman, M.A.; Law, S.E.; J. E.nviron. Eng-ASCE 1998,124, 272.

95. Lopez, A.; Ricco, G.; Ciannarella, R.; Rozzi, A.; Di Pinto, A.C., Passino, R.; Wat. Sci. Technol. 1999, 40, 99.

96. O’Neill, C.; Lopez, A.; Esteves, S.; Hawkes, D.L.; Wilcox, S.; Apl. Microbiol. Biotechnol. 2000, 53, 249.

97. Kunz, A.; Reginatto, V.; Duran, N.; Chemosphere 2001, 44, 281.

98. Palma, C.; Moreira, M.T.; Mielgo, I.; Feijo, G.; Lema, J.M. Wat. Sci. Technol. 1999, 40, 131. 